\title{
Heart glucose transport and transporters in rat heart: regulation by insulin, workload and glucose
}

\author{
D.Zaninetti, R. Greco-Perotto and B.Jeanrenaud \\ Laboratoires de Recherches Métaboliques, Faculty and Department of Medicine of Geneva, Geneva, Switzerland
}

Summary. Aspects of the regulation of the glucose transport by perfused hearts of normal rats have been studied by measuring glucose transport (via the efflux of labelled 3-O-methyl-D-glucose) and glucose transporters (via the labelled cytochalasin B binding assay). Similary to what is observed with insulin, increasing workload (by raising perfusion pressure from 50 to $100 \mathrm{~mm} \mathrm{Hg}$ ) stimulated glucose transport 7 to 8-fold. Glucose (via its analog 3-O-methylglucose, used at $15 \mathrm{mmol} / 1)$ stimulated its own transport 4-fold. The three stimuli favored the translocation of glucose transporters from an intracellular pool (microsomes) to the plasma membrane. Insulin increased the apparent affinity (decreased dissociation constant values) of plasma membrane transporters for cytochalasin, as well as the Hill coefficient, indicating the oc- currence of a positive cooperativity amongst plasma membrane transporters. Workload increased only the Hill coefficient, glucose only the apparent affinity for cytochalasin of plasma membrane transporters. This study shows that insulin, workload and glucose itself stimulate glucose transport by favouring the translocation process of glucose transporter as well as by changing, albeit by a different mechanism, the functional properties of the transporters once translocated to the plasma membrane.

Key words: Perfused hearts, glucose transport, glucose transporters, cytochalasin B binding assay, insulin effect, workload effect, glucose effect.
It has been shown previously that insulin stimulates glucose uptake in isolated adipocytes by favouring the translocation of glucose transporters from an intracellular pool (microsomes) to the plasma membrane [1]. The same process has been subsequently shown to occur in the muscle (diaphragm) [2]. More recently, it was demonstrated by using normal rat brown adipose tissue $[3,4]$ or perfused normal rat hearts [5], that, although the translocation process was a fundamental property of the glucose transport system, it did not account for all the changes observed. In particular, both in brown adipose tissue [4] and the heart [5], insulin activated the transporters once translocated into the plasma membrane. This activation was evidenced by insulin-induced increases in the affinity of the plasma membrane transporters for cytochalasin and in the Hill coefficient [6] thereof, indicative of the possible presence of a positive cooperativity amongst transporters [3-5].

In muscles, the study of glucose transport and, to a greater extent, that of the glucose transporter system, is hampered by technical difficulties. Thus, the preparation of purified muscle plasma and microsomal mem- branes in amounts sufficient to measure glucose transporters by the labelled cytochalasin $\mathrm{B}$ binding requires a large quantity of tissue. Although the heart is barely representative of muscle mass it is, for the moment, the only preparation that actually permits measurements of both glucose transport and glucose transporters. In spite of this limitation, conclusions drawn from studies carried out with this organ are potentially interesting and applicable to other types of muscles, at least until other technological approaches make it possible to investigate other muscle types.

It has been reported that insulin and workload (achieved by increasing perfusion pressure) are two important factors in regulating glucose uptake by normal hearts [7]. The rise in aortic pressure, e.g. from 50 to $100 \mathrm{~mm} \mathrm{Hg}$, has been shown to increase oxygen consumption by approximately 2.5 -fold in hearts perfused with a medium containing glucose without insulin, and to increase glucose uptake as well as glycolysis [7]. It has also been reported, in rat epitrochlearis muscle, that insulin or increasing contractile activity stimulates glucose transport and metabolism via independent mechanisms [8]. Thus, it has been demonstrated that 
increased contractile activity accelerated the rate of glucose transport even in insulin deficient skeletal muscles [8].

Besides insulin and workload, a role for glucose in the regulation of hexose transport has been suggested in adipocytes [9]. It has been shown that glucose is involved in the regulation of its own transport in the 3T3 adipocytes, as the hexose modulated the rate of degradation, internalisation or inactivation of its specific transporters [10]. It has also been postulated that glucose could "up-and-down" regulate its own transport, presumably by changing the number of glucose transporters present in the plasma membrane of skeletal muscle [11].

Previous studies from this laboratory have shown that insulin, when added to perfused hearts of normal rats, stimulated D-glucose transport [5]. They also showed (upon measuring glucose transporters in enriched plasma and microsomal membranes) that the stimulatory effect of insulin on the hexose transport was due not to the sole translocation of intracellular glucose transporters to the plasma membrane, but to changes in the functional properties thereof, notably and possibly in the occurrence of a positive cooperativity amongst plasma membrane transporters [5].

Due to this, the aim of the present study was to define the respective effect of workload, or glucose itself on 3-O-methyl-D-glucose transport (the non-metabolised analog of D-glucose) in perfused normal rat hearts, and to attempt to define the effects of these factors on the heart D-glucose transporter system.

\section{Materials and methods}

\section{Animals}

Fifteen-week-old normal male rats of the Zucker strain (FA/FA) bred in our laboratory were used. They had free access to a standard laboratory chow (Lacta 299, Provimi-Lacta SA, Cossonay, Vaud, Switzerland) and were maintained at a constant temperature $\left(22^{\circ} \mathrm{C}\right)$ in an animal quarter with a $12-\mathrm{h}$ artificial light cycle.

\section{Heart perfusion and 3-O-methylglucose transport studies}

Rats were anaesthetised with pentobarbital $\left(90 \mathrm{mg} \cdot \mathrm{kg}^{-1}\right)$. The heart was rapidly removed, placed in an ice-cold $0.9 \% \mathrm{NaCl}$ solution for $10 \mathrm{~s}$, cannulated via the aorta and perfused according to Langendorff $[7,12]$. The perfusion medium consisted of a Krebs Ringer bicarbonate buffer, $\mathrm{pH} 7.4$, and pyruvate $(5 \mathrm{mmol} / 1)$ as energy source [13]. For measuring 3-O-methyl-D-glucose (3-O-M-glucose) transport, hearts were preperfused for $10 \mathrm{~min}$ with or without the stimuli defined below, with a recirculating medium containing $6 \mathrm{mmol} / 1$ $(0.3 \mathrm{mCi} / \mathrm{l}){ }^{14} \mathrm{C}-3-\mathrm{O}-\mathrm{M}$-glucose and $6 \mathrm{mmol} / 1(0.3 \mathrm{mCi} / \mathrm{l}){ }^{3} \mathrm{H}-\mathrm{L}-\mathrm{glu}-$ cose (loading period). Ten minutes were needed to reach steady-state levels of labelled substrates. Labelled L-glucose was used to calculate the extracellular and free diffusion components. At the end of the loading period, perfusion was continued without recirculation, while switching to a medium containing no sugar. Aliquots of the medium were then collected every $12 \mathrm{~s}$ in vials placed on a fraction collector (Gilson TDC 220, Villier le Bel, France). Each vial contained $12 \mathrm{~s}$ fractions of the medium with 3-O-M-glucose and L-glu- cose arising from the heart. 3-O-M-glucose was in the intra- and the extracellular space; L-glucose mainly in the extracellular and, to some extent (via simple diffusion), in the intracellular space. 3-O-Mglucose counter-transported by facilitated diffusion (efflux) could be calculated by subtracting, in each vial, total labelled L-glucose content from total labelled 3-O-M-glucose content. The plot of cumulative effluxes as a function of time was used to obtain kinetics of efflux. Various tests of linearity (e.g. 0, 1,2 order kinetics) indicated that cumulative efflux curves fitted the first order kinetic formula: $A(t)=A_{0}\left(1-e^{-k t}\right)$, where $A$ was the quantity of effluent hexose(s) at given time $(t) ; A_{0}$ the maximal quantity of effluent hexose at infinite time; $e=$ base of natural logarithm; $k=$ the kinetic constant. The rate of efflux (V) during a small period of time was: $\mathrm{V}=\mathrm{dA} / \mathrm{dt}=\mathrm{kA} \mathrm{A}_{0} \mathrm{e}$ (kt). As initial rate of efflux mimicked glucose transport, the rate was calculated with a $t$ value equal to $0 \mathrm{~s}$. The initial speed $\left(\mathrm{V}_{\mathrm{i}}\right)$, thus, was: $\mathrm{V}_{\mathrm{i}}=\mathrm{kA}_{0}$, expressed as $\mu \mathrm{mol} \cdot \mathrm{min}^{-1} \cdot \mathrm{heart}^{-1}$. Unless stated otherwise, hearts were perfused at low pressure $(50 \mathrm{~mm} \mathrm{Hg})$, a situation in which the effect of insulin on glucose uptake is maximal [14]. When basal 3-O-methyl-D-glucose efflux rates were investigated a pre-perfusion period of $10 \mathrm{~min}$ was allowed to wash away endogenous insulin. In some experiments, the effect of insulin was reavaluated and the hormone $(10 \mathrm{U} / 1)$ was added to both pre- and perfusion media. The workload effect on glucose efflux rates was measured by raising the perfusion pressure (during the pre- and perfusion periods) from 50 to $100 \mathrm{~mm} \mathrm{Hg}$. The effect of D-glucose on its own transport was investigated by adding to the perfusion medium (during the pre- and perfusion periods) $15 \mathrm{mmol} / 1$ of 3-O-methyl-Dglucose, with $15 \mathrm{mmol} / \mathrm{l} \mathrm{L-glucose}$ as extracellular marker.

\section{Heart plasma and microsomal membrane preparation}

For membrane preparation, hearts were perfused for $15 \mathrm{~min}$ in the presence or absence of stimulus indicated above. Hearts were then disconnected from the perfusion apparatus and immediately frozen in liquid nitrogen. Frozen hearts were stored at minus $70^{\circ} \mathrm{C}$. The membrane preparations (respectively plasma and microsomal membranes) were then carried out as recently described elsewhere [5].

\section{Enzyme marker assays}

Total proteins were measured by the Bio-Rad method, with gamma globulin as standard [15]. The 5'nucleotidase was the marker used for plasma membranes and was determined as described elsewhere [16]. The NADPH cytochrome $c$ reductase, a marker of microsomal membranes, was determined according to the method of Sottocasa et al. [17].

\section{Cytochalasin B binding assays}

Cytochalasin B binding was carried out on plasma and microsomal membranes obtained as mentioned above, initially using ten concentrations of the ligand $(40-500 \mathrm{nmol} / 1)$. As binding curves obtained with either 10 or 5 different ligand concentrations resulted in identical results, subsequent experiments were made with only five ligand concentrations [3-5]. As also described previously [4, 5], binding of labelled cytochalasin was carried out either in the presence of $250 \mathrm{mmol} / 1$ sucrose (i. e. specific + unspecific bindings) or in that of $500 \mathrm{mmol} / 1$ unlabelled glucose (non-specific binding). The differences between the two binding curves yielded the specific (i.e. D-glucose displaceable) cytochalasin B. It has been shown $[4,5]$ that at such viscosity even $1 \mathrm{~h}$ of ultracentrifugation at $436,000 \mathrm{~g}$ in a TL 100 centrifuge (Beckman, Fullerton, Calif, USA) failed to totally pellet the membrane fraction, about $25 \%$ protein still being in the supernatant. Due to this, the initial cytochalasin B method [1] was modified and validated [3-5], by precipitating all duplicate $(50 \mu \mathrm{l})$ samples of each of the final supernatants with $10 \mu \mathrm{l} 0.15 \mathrm{~mol} / 1$ barium hydroxide, followed by $10 \mu 10.15 \mathrm{~mol} / 1$ zinc sulfate. This precipitated all proteins, including the glucose transporters. The modified method does not alter binding properties as demonstrated elsewhere [3-5]. 
Due to the modification of the labelled cytochalasin B binding assay mentioned above, the Hill equation [6] was used to analyse the data as specified elsewhere [3-5]. The plot of the linearised Hill equation, i.e. $\log (\mathrm{b} / \mathrm{Ro}-\mathrm{b})=n \log (\mathrm{F})-\log \left(\mathrm{K}^{\prime}\right)$ (in which $\mathrm{Ro}=$ maximal binding capacity; $\mathrm{b}=$ bound cytochalasin $\mathrm{B} ; \mathrm{F}=$ free cytochalasin $\mathrm{B} ; n=$ Hill coefficient $; \mathrm{K}^{\prime}=$ constant comprising interaction factor and intrinsic dissociation constant; $\mathrm{Kd}=\mathrm{K}^{\prime / \mathrm{n}}=$ dissociation constant) permitted us to obtain the Hill coefficient $n$, the Ro value (maximal binding) and normalised Kd values. The Ro value was found by a program that iterates calculations with increasing Ro values until the maximal coefficient of correlation was reached; $\mathrm{n}$ was the slope of the linearised Hill equation and $\mathrm{Kd}=10^{\log (\mathrm{F})}$ where $\log (\mathrm{b} / \mathrm{Ro}-\mathrm{b})=0$. Using the values of $n, \mathrm{Kd}$ and Ro, the Hill curve was plotted (bound versus free) together with the data points. In the present paper, such a plot is represented only to revalidate the methodology used (insulin data), the remaining results being, for clarity sake, shown as table $(n, \mathrm{Kd})$ and bar graphs (Ro).

\section{Statistical analysis}

Statistical analyses were performed with the two-tailed Student's t-test for unpaired data. The $2 p$ values obtained indicate the probability that the two events are similar.

\section{Results}

\section{3-O-methyl-D-glucose transport}

The 3-O-methyl-D-glucose (3-O-M-glucose) transport was measured by its efflux from preloaded hearts. Under basal conditions (i.e. at $6 \mathrm{mmol} / 1$ 3-O-M-glucose, $50 \mathrm{~mm} \mathrm{Hg}$ perfusion pressure, no insulin) and as illustrated by Figure 1, 3-O-M-glucose transport was $4.2 \pm$ $0.2 \mu \mathrm{mol} \cdot \mathrm{min}^{-1} \cdot g$ dry weight ${ }^{-1}$. As further illustrated by Figure 1, 3-O-M-glucose transport was stimulated by increased workload as well as by 3-O-M-glucose itself, each effector being used at its maximal stimulatory effect. The workload effect on 3-O-M-glucose transport was similar to that seen with a maximal insulin concentration [5], while a more moderate stimulation of glucose transport was elicited by adding its analog 3-O-methyl-glucose.

\section{Cytochalasin B binding}

The D-glucose inhibitable cytochalasin binding assays were performed on enriched plasma and microsomal membranes obtained from perfused hearts. The enrichment of plasma and microsomal membrane was similar to that previously reported [5]. It was not modified by the experimental conditions and was, respectively, close to 18-fold for plasma (with 5'nucleotidase used as enzyme marker), and 7.5-fold for microsomal membranes (with NADPH cytochrome $\mathrm{c}$ reductase used as enzyme marker). The recoveries (expressed as percentages of values found in the initial homogenates) were $19.3 \pm 0.9$ for plasma membranes and $24.4 \pm 1.2$ for microsomal ones (means $\pm S E M$ of all membrane preparations). Recoveries of membrane proteins were also found to be constant, being $2.6 \pm 0.1$ and $1.4 \pm$ $0.2 \%$ of initial homogenate, for plasma and microsom-

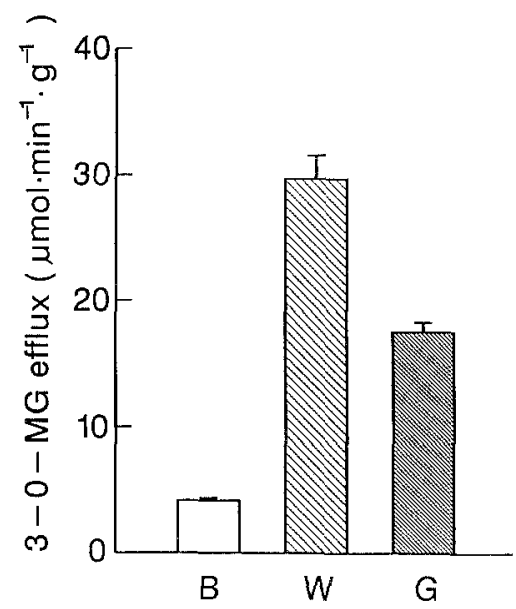

Fig. 1. Effect of workload or glucose itself on 3-O-methyl-glucose (3-O-MG) (G) transport by perfused normal rat hearts. 3-O-MG transport was measured by its efflux from preloaded organs, under basal (B) conditions (50 $\mathrm{mm} \mathrm{Hg}$ perfusion pressure); following increased workload (W) achieved by raising perfusion pressure (from 50 to $100 \mathrm{~mm} \mathrm{Hg}$ ); following addition of a high $(15 \mathrm{mmol} / \mathrm{l}) 3-\mathrm{O}-$ $\mathrm{MG}$ concentration. For comparison, note that maximal insulin concentration $(10 \mathrm{U} / \mathrm{l})$ augmented the hexose transport to $31.8 \pm 2.6$. Values are means of 4-6 experiments \pm SEM. Stimulated conditions are statistically different from basal (B), with workload $2 p<0.002$; glucose $2 p<0.001$

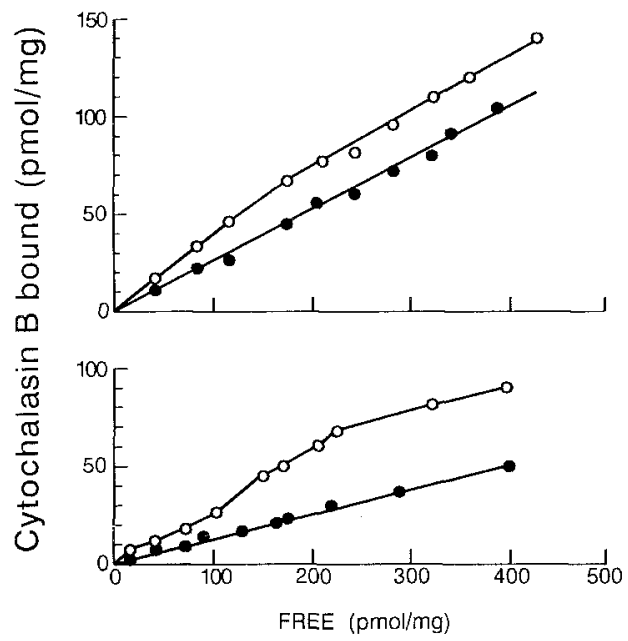

Fig. 2. $\left[{ }^{3} \mathbf{H}\right]$ Cytochalasin $\mathbf{B}$ binding sites in plasma membranes from hearts perfused with or without $10 \mathrm{U} / 1$ insulin. $\left[{ }^{3} \mathrm{H}\right]$ Cytochalasin $B$ binding to plasma membranes from control (upper panel) and insulin-stimulated (lower panel) hearts has been measured at 10 different concentrations of the ligand in the absence $(O)$ or presence $(0)$ of $500 \mathrm{mmol} / 1$ unlabelled D-glucose as described under "Materials and methods". Results are plotted as bound versus free cytochalasin and were calculated according to Hill. Upper panel: Ro $24.6 \mathrm{pmol} / \mathrm{mg}$ membrane protein; Hill coefficient $(n) 1.49 ; \mathrm{Kd} 208 \mathrm{nmol} / 1$. Lower panel: Ro $41.2 \mathrm{pmol} / \mathrm{mg}$ membrane protein, Hill coefficient $(n) 2.16$; $\mathrm{Kd} 98 \mathrm{nmol} / \mathrm{l}$. Points are mean of one experiment. Ro=maximal binding, $n=$ slope of linearised Hill equation, $\mathrm{Kd}=$ dissociation constant (see "Materials and methods")

al membranes respectively (means \pm SEM of all membrane preparations). The measured specific cytochala$\sin \mathrm{B}$ binding activities have been expressed as pmol/ $\mathrm{mg}$ of protein and adjusted to those which would have been observed, had the membrane fractions been free 


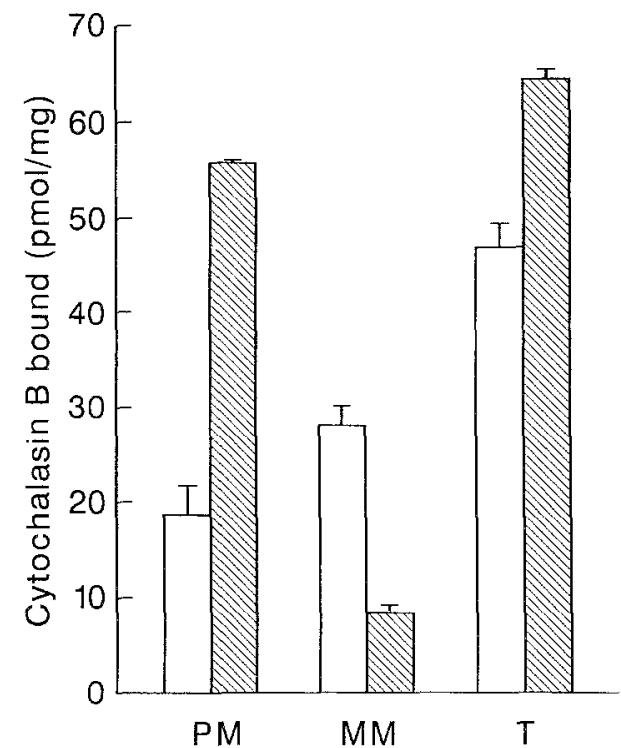

Fig. 3. Effect of increased workload on the number of D-glucose transporters (measured by the specific binding of labelled cytochala$\sin \mathrm{B}$ ) in plasma (PM) and microsomal (MM) membranes from perfused normal rat hearts. Increased workload ( was achieved by raising perfusion pressure from $50 \mathrm{~mm} \mathrm{Hg}$ (controls, open bars) to $100 \mathrm{~mm} \mathrm{Hg}$. Total $(\mathrm{T})$ refers to the number of glucose transporters present in both plasma (PM) and microsomal (MM) membranes. Each bar is the mean \pm SEM of 3 individual experiments. Increased workload effect on MM and PM glucose transporter number: $2 p$ at least $<0.01$. Increased workload effect on T: $2 p<0.01$

Table 1. Effect of workload or glucose on the constant of dissociation $(\mathrm{Kd})$ and the Hill coefficient of the glucose transporter for the cytochalasin B of plasma and microsomal membrane, obtained from perfused hearts of normal rats

\begin{tabular}{lccc}
\hline & Controls & $\begin{array}{l}\text { Increased } \\
\text { workload }\end{array}$ & Glucose \\
\hline Hill coefficient $(n)$ & & & \\
Plasma membrane & $1.4 \pm 0.2$ & $2.3 \pm 0.1^{\mathrm{a}}$ & $1.8 \pm 0.1$ \\
Microsomes & $2.2 \pm 0.2$ & $2.2 \pm 0.4$ & $2.2 \pm 0.1$ \\
$K d$ & & & \\
Plasma membrane & $272 \pm 60$ & $192 \pm 11$ & $166 \pm 11^{\mathrm{a}}$ \\
Microsomes & $187 \pm 16$ & $267 \pm 24$ & $164 \pm 41$ \\
\hline
\end{tabular}

Values are means \pm SEM of at least 3 individual experiments. The dissociation constant $(\mathrm{Kd})$ and Hill coefficient (n) were determined from the Hill analysis of the cytochalasin B binding curves (see Material and methods). Controls: hearts perfused at $50 \mathrm{~mm} \mathrm{Hg}$; increased workload was achieved by raising perfusion pressure from 50 to $100 \mathrm{~mm} \mathrm{Hg}$; glucose stimulation was obtained by adding 3-Omethyl-D-glucose $(15 \mathrm{mmol} / \mathrm{l})$ to hearts. ${ }^{a}$ Indicates values from stimulated conditions that are statistically different from control, with at least $2 p<0.05$

of cross-contamination. Such adjustments have been based on specific activities of the enzyme markers and protein recoveries, with the assumption that 5 'nucleotidase activity is localised specifically in plasma membranes and that NADPH cytochrome c reductase activity is specific for microsomal membrane. It should be emphasised that each sample was always corrected for the cross-contamination just mentioned, a contamination that was, as a mean, $10 \%$ plasma membranes in

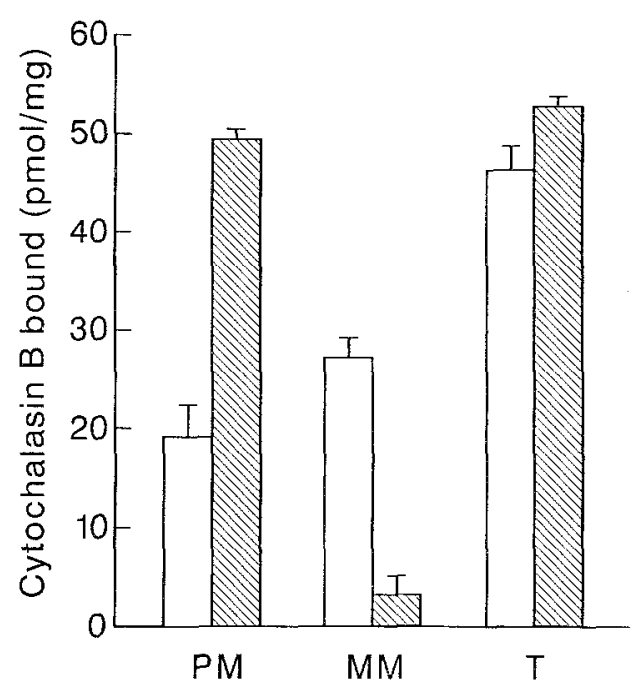

Fig. 4. Effect of unlabelled 3-O-methyl-D-glucose on the number of D-glucose transporters (measured by the specific binding of labelled cytochalasin B) in plasma (PM) and microsomal (MM) membranes from perfused normal rat hearts. When present, 3-O-methyl-D-glucose was at a concentration of $15 \mathrm{mmol} / \mathrm{l}$. Total (T) refers to the number of glucose transporters present in both plasma (PM) and microsomal membranes (MM). Each bar is the mean \pm SEM of 3 individual experiments. Controls (open bars) were hearts perfused with $6 \mathrm{mmol} / \mathrm{l}$ 3-O-methyl-D-glucose. Hexose effect on $\mathrm{MM}$ and PM glucose transporter number: $2 p$ at least $<0.01$

microsomes and 40-45\% microsomal membranes in the plasma ones.

The cytochalasin $\mathrm{B}$ binding assay was first reassessed using plasma membranes obtained from hearts perfused with or without insulin, and ten different ligand concentrations (Fig. 2). The binding curves so obtained were similar, for plasma membranes, to those obtained previously with five ligand concentrations [5]. As can be seen, $24.6 \mathrm{pmol} / \mathrm{mg}$ protein glucose transporters were measured in the basal state and $41.2 \mathrm{pmol} / \mathrm{mg}$ protein when insulin was present. Insulin favoured, as published elsewhere [5], the translocation of glucose transporter from microsomal to plasma membranes. Furthermore, insulin changed (Fig. 1) the properties of the glucose transporters present in the plasma membrane: the dissociation constant of cytochalasin B binding decreased from $208 \mathrm{nmol} / 1$ under basal conditions to $98 \mathrm{nmol} / 1$ in the presence of insulin. The Hill coefficient, an index of cooperativity between transporters for cytochalasin B, augmented in plasma membranes from a basal value of 1.5 (control hearts) to 2.2 (insulin-treated hearts). Data obtained with ten (this study) or five ligand concentrations [5] being identical, subsequent cytochalasin $\mathrm{B}$ assays were made using the latter conditions.

The increase in workload (from 50 to $100 \mathrm{~mm} \mathrm{Hg}$ perfusion pressure) had an insulin-like effect upon the redistribution of the glucose transporters between the microsomal and the plasma membranes. It favoured, as shown by Figure 3, the appearance of transporters 
(mean of 3 individual experiments) in the plasma membranes while decreasing those present in micro-

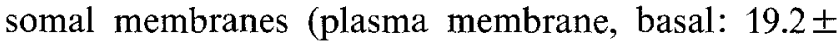
3.2; increased workload: $56.3 \pm 0.3$; microsomes, basal: $22.5 \pm 5.0$; increased workload: $9.6 \pm 0.9 \mathrm{pmol} / \mathrm{mg}$ protein). In contrast to insulin and as published elsewhere [5], the total number of transporters (plasma plus microsomal) was increased by workload to a value of $66 \pm 1$ compared to a basal value of $46.4 \pm 2.4 \mathrm{pmol} /$ $\mathrm{mg}$ protein (Fig.3). Increased workload partly modified, as illustrated by Table 1, the properties of the glucose transporters present in the plasma membranes. Thus, the dissociation constant of the cytochalasin B binding decreased from $272 \pm 60 \mathrm{nmol} / 1$ (basal conditions) to $192 \pm 11$ when workload was increased, a change that failed to reach statistical significance. Increased workload also provoked the occurrence of a positive cooperativity amongst plasma membrane transporters, as evidenced by the Hill coefficient for cytochalasin B which increased from a basal value of $1.4 \pm 0.2$ to $2.3 \pm 0.1(2 p<0.002)$.

D-glucose, as tested only via its non-metabolised analog 3-O-M-glucose used at $15 \mathrm{mmol} / \mathrm{l}$ concentration had insulin-like effect upon the translocation process of glucose transporters, as illustrated by Figure 4 . Indeed, $49.5 \pm 1.0 \mathrm{pmol} / \mathrm{mg}$ protein glucose trans :ters were present (mean of 3 individual experim ,) in the plasma membrane following perfusions with 3-O-M-glucose, compared to a basal number of transporter of $19.2 \pm 3.2$. Concomitantly, the number of microsomal glucose transporters decreased from $27.2 \pm$ 2.0 (basal state) to $3.2 \pm 1.9$ in hearts perfused with the analog of D-glucose. The total number of transporters remained unchanged in both conditions $(46.4 \pm 2.4$, basal, $52.7 \pm 1.0$ plus $3-\mathrm{O}$-M-glucose $\mathrm{pmol} / \mathrm{mg}$ protein, NS). As described in Table 1, 3-O-M-glucose failed to change the Hill coefficient of plasma membrane glucose transporters for cytochalasin while it significantly decreased the dissociation constant of cytochalasin B binding when compared to basal (i.e. no added insulin) conditions.

\section{Discussion}

As the rate of glucose transport into muscles is thought to be a rate limiting step of its subsequent utilisation [18], the mechanisms that regulate this process are important to delineate. The aim of the present study was to amplify previous data showing that insulin stimulates 3-O-methyl-glucose transport, an effect that appears to be due not to the sole translocation of intracellular transporters to the plasma membrane, but to changes in the functional properties thereof [5]. To do so, the actual D-glucose transport was measured, as was the state of glucose transporters, under the influence of two insulin unrelated stimuli (i.e. increased workload and D-glucose itself), using as a tool the perfused heart from normal rats.
As the transport of D-glucose in the heart has been shown to be symmetrical [18], D-glucose uptake was assessed by the rate of efflux of its non-metabolised analog, the 3-O-methyl-D-glucose (3-O-M-glucose). The glucose transporter system was studied by the labelled cytochalasin B assay, as recently modified and validated [3-5]. The specific cytochalasin B binding curves (those which represent specific binding to the glucose transporter molecules) were analysed according to Hill [6] to determine, not only the number of transporters present either within the plasma or microsomal membranes, but also the properties thereof, i.e. the dissociation constant of the cytochalasin binding $(\mathrm{Kd})$ and the Hill coefficient $(n)$. The latter, when reaching values greater than 1 , indicate the likely appearance of a positive cooperativity amongst glucose transporters.

With regard to D-glucose transport, this study shows that not only insulin [5], but increasing workload (perfusion pressure of the heart raised from 50 to $100 \mathrm{~mm} \mathrm{Hg}$ ) stimulates the hexose transport in normal rat hearts by 7 to 8 -fold compared to the basal values [5]. The uptake of D-glucose is also stimulated by itself (when using its analog 3-O-methyl-glucose at the high concentration of $15 \mathrm{mmol} / 1$ as stimulus), although the stimulatory effect is about half that seen with either insulin [5], or increasing workload (Fig. 1).

When insulin was added to perfused hearts, the hormone increased the number of plasma membrane glucose transporters as previously reported [5] and confirmed in this study (Fig. 2). This was achieved by insulin favouring translocation of intracellular transporters from an intracellular pool to the plasma membrane as described elsewhere $[1,2,4,5]$. Insulin also altered cytochalasin B binding properties of transporters once in the plasma membrane (increased Hill coefficient, decreased Kd values, Fig. 2).

The novelty of the present study is to show that the translocation of glucose transporters from microsomal to the plasma membranes is not restricted to insulin [5], but is shared by at least two other seemingly unrelated stimuli, workload and D-glucose (Figs.3-4). It thus appears that the three stimuli tested in perfused hearts (i.e. insulin, workload, glucose itself) do not operate precisely by the same mechanism(s), although they all stimulate 3-O-methyl-D-glucose uptake.

The number of glucose transporters present within the plasma membrane doubled under the effect of insulin. Furthermore, as previously published [5], the hormone decreased the Kd value of the plasma membrane transporters for cytochalasin B, thereby increasing the affinity of those transporters for ligand (possibly for glucose). It also increased the Hill coefficient of plasma membrane glucose transporters for cytochala$\sin \mathrm{B}$ (from 1.4 to $2.7,2 p<0.002$ ), thereby possibly favouring the appearance of a positive cooperativity among these transporters. Thus, these and previous data [5] suggest that insulin, in addition to favouring the 
translocation process of glucose transporters, may activate the latter once present in the plasma membrane (Fig. 2).

Workload differs from insulin in increasing to a greater extent (about 3-fold instead of two) the number of glucose transporters within the plasma membrane. It also appears to recruit, by as yet unknown mechanisms, masked transporters whose total number increases under its influence. No protein inhibitor was used in this study to rule out the possibility that workload increased the de novo synthesis of transporters. The short $(15 \mathrm{~min})$ experimental time used for testing the increased workload effect makes the occurrence of a de novo synthesis process unlikely. Workload does not modify the apparent affinity of the plasma membrane transporters for cytochalasin $\mathrm{B}$, but it increases the Hill coefficient to the same extent as insulin (Table 1). Thus, increased workload has an insulin-like effect on D-glucose transport (Figs. 1, 3) but it acts by somewhat different mechanisms than the hormone. It apparently recruits and translocates more transporters to the plasma membranes than insulin, then activates them, albeit by other means than insulin (Figs. 2, 3, Table 1).

Glucose favours the process of transporter translocation, thereby increasing plasma and decreasing microsomal membrane glucose transporters (Fig. 4). Although glucose does not "activate" plasma glucose transporters (no change in Hill coefficient), it increases the apparent affinity of the plasma membrane transporters for cytochalasin B (thereby possibly for glucose, Table 1).

In summary, insulin, increased workload, and glucose itself, all stimulate D-glucose uptake. All stimuli favour a translocation process of transporters, increasing the number of glucose transporters within the plasma membrane decreasing that present in microsomal ones. As previously reported [5], insulin alters the functional properties of plasma membrane glucose transporters (increased Hill coefficient, increased affinity for cytochalasin B). Workload or glucose itself change only one of these two parameters, the former increasing Hill coefficient, the latter the affinity of the plasma membrane transporters for cytochalasin.

These data together suggest that in vivo, when exposed to the simultaneous stimulatory effect of insulin, workload, and glucose, glucose uptake by the heart (possibly by other muscles) must be the result a series of complex events that cannot be accounted for solely by the translocation process of glucose transporters, but comprises a modulation in the intrinsic activity of the transporters once in present in the plasma membrane.

Acknowledgements. This work has been supported by Grant No 3.822.086 from the Swiss National Science Foundation (Berne, Switzerland) and by a grant-in-aid from Nestlé S. A. (Vevey, Switzerland). We thank Ms Karin Petermann for her excellent secretarial help.

\section{References}

1. Cushman SW, Wardzala LJ (1980) Potential mechanism of insulin action on glucose transport in the isolated rat adipose cell. Apparent translocation of intracellular transport systems to the plasma membrane. J Biol Chem 255: 4758-4762

2. Wardzala LJ, Jeanrenaud B (1981) Potential mechanism of insulin action on glucose transport in the isolated rat diaphragm. $J$ Biol Chem 256: 7090-7093

3. Greco-Perotto R, Zaninetti D, Assimacopoulos-Jeannet F, Bobbioni E, Jeanrenaud B (1987) Stimulatory effect of cold adaptation on glucose utilisation by brown adipose tissue. J Biol Chem 262: 7732-7736

4. Greco-Perotto R, Assimacopoulos-Jeannet F, Jeanrenaud B (1987) Insulin modifies the properties of glucose transporters in rat brown adipose tissue. Biochem $\mathrm{J} 247$ : 63-68

5. Zaninetti D, Greco-Perotto R, Assimacopoulos-Jeannet F, Jeanrenaud B (1988) Effects of insulin on glucose transport and transporters in rat heart. Biochem J 249 (in press)

6. Hill AV (1910) The possible effects of the aggregation of the molecules of haemoglobin on its dissociation curves. J Physiol (London) 40: 190-229

7. Neely JR, Liebermeister H, Battersby EJ (1967) Effect of pressure development on oxygen consumption by isolated heart. Am J Physiol 212: 804-814

8. Nesher R, Karl IE, Kipnis DM (1985) Dissociation of effects of insulin and contraction on glucose transport in rat epitrochlearis muscle. Am J Physiol 249: C226-C232

9. Ciaraldi TP, Kolterman OG, Siegel JA, Olefsky JM (1979) Insulin stimulated glucose transport in human adipocytes. Am J Physiol 236: E621-E625

10. Van Putten JPM, Krans HMJ (1985) Glucose as a regulator of insulin-sensitive hexose uptake in 3T3 adipocytes. J Biol Chem 260: 7996-8001

11. Sasson S, Cerasi E (1986) Substrate regulation of the glucose transport system in rat skeletal muscle. J Biol Chem 261: $16827-16833$

12. Taegtmeyer H, Hems R, Krebs HA (1980) Utilisation of energyproviding substrates in the isolated working rat heart. Biochem J 186: 701-711

13. Crettaz M, Prentki M, Zaninetti D, Jeanrenaud B (1980) Insulin resistance in soleus muscle from obese Zucker rats. Involvement of several defective sites. Biochem J 186: 525-534

14. Neely JR, Denton RM, England PJ, Randle PJ (1972) The effects of increased heart work on the tricarboxylate cycle and its interactions with glycolysis in the perfused rat heart. Biochem $\mathbf{J} 128$ : 147-159

15. Bradford $M$ (1976) A rapid and sensitive method for the quantitation of microgram quantities of protein utilizing the principle of protein-dye binding. Anal Biochem 72: 248-254

16. Avruch J, Wallach DFH (1971) Preparation and properties of plasma membrane and endoplasmic reticulum fragments from isolated rat fat cells. Biochem Biophys Acta 233: 334-347

17. Sottocasa GL, Kuylenstierna B, Ernster L, Bergstand A (1967) An election transport system associated with the outer membrane of liver mitochondria. A biochemical and morphological study. J Cell Biol 32: 415-438

18. Zaninetti D, Crettaz M, Jeanrenaud B (1983) Dysregulation of glucose transport in hearts of genetically obese $(\mathrm{fa} / \mathrm{fa})$ rats. Diabetologia $25: 525-529$

Received: 21 August 1987

and in revised form: 10 December 1987

Prof. B. Jeanrenaud

Laboratoires de Recherches Métaboliques

Avenue de la Roseraie 64

CH-1211 Geneva 4

Switzerland 